\title{
The association between sarcopenia and quality of life is different in community dwelling older Australian men and
} women

\author{
Tsung Woo ${ }^{1,2,3}$, Solomon $\mathrm{Yu}^{1,2,3 *}$, Robert Adams ${ }^{4}$ and Renuka Visvanathan ${ }^{1,2,3}$ \\ ${ }^{1}$ National Health and Medical Council Centre of Research Excellence: Trans-Disciplinary Frailty Research to Achieve Health Aging, Australia \\ ${ }^{2}$ Adelaide Geriatrics Training and Research with Aged Care (GTRAC) Centre, School of Medicine, Faculty of Health Sciences, University of Adelaide, Australia \\ ${ }^{3}$ Aged \& Extended Care Services, The Queen Elizabeth Hospital, Central Adelaide Local Health Network, Australia \\ ${ }^{4}$ Health Observatory, School of Medicine, Faculty of Health Sciences, University of Adelaide, Australia
}

\begin{abstract}
Background: The impact of sarcopenia on health-related quality of life (HRQoL) in community dwelling older people in Australia is not known. The aim of this study was to evaluate the relationship between sarcopenia and HRQoL in older Australian men and women.

Methods: 357 men and 370 women aged 65 years and older from the North West Adelaide Health Study at Stage 2 (2002-2004). Sarcopenia was defined as the presence of low muscle mass and low grip strength. HRQoL was assessed using the Short Form-36 (SF-36) questionnaire: physical component summary (PCS) and mental component summary (MCS). Analyses were performed using multiple regression and adjustments were made for age, physical activity, smoking status, co-morbidity and depression.
\end{abstract}

Results: The prevalence of sarcopenia was $10.1 \%$ in men and $9.5 \%$ in women. Men with sarcopenia was significantly associated with the PCS score in the unadjusted model $(\mathrm{P}=0.012)$ and only model 1 adjusted for age $(\mathrm{P}=0.041)$. No significant association was noted in model 2 (model $1+$ physical activity and smoking status) and model 3 (model 2 + Charlson co-morbidity index and depression). In men, a significant association between sarcopenia and MCS score was seen in the unadjusted and all 3 adjusted models. No association was seen between sarcopenia and the PCS or MCS score in women for both the adjusted and unadjusted models.

Conclusion: After adjusting for multiple confounders, the association between sarcopenia only remained for the MCS score, in men. No association between sarcopenia and HRQoL was seen in women.

\section{Introduction}

Sarcopenia, the age-associated loss of skeletal muscle mass and function [1] is as a result of multiple interacting intrinsic (i.e. chronic inflammatory states) and extrinsic (i.e. decreased physical activity) factors [2]. Sarcopenia is also an independent risk factor for adverse health outcomes and is not limited to disability $[3,4]$, mortality $[5,6]$, falls and fractures and hospitalization [7]. In one American study, the impact of sarcopenia on direct health care cost was staggering at $\$ 18$ billion in 2000 [8]. The same study postulated that a $10 \%$ reduction in sarcopenia prevalence through interventions that maintain or slow the progression of the loss of muscle mass, such as adequate protein intake and increased physical activity coupled with resistance exercise could potentially save $\$ 1.1$ billion per year in healthcare costs [8]. Sarcopenia affects one in five older people aged 80 years and over and with population ageing, the health burden arising from sarcopenia will only increase if left unaddressed [9]. Doing nothing is not an option as this will be unaffordable.

In older age, it is about the quality of life rather than the quantity of life. Quality of life to an older person is the ability to achieve a contented, meaningful, and fulfilling life [10]. Health related quality of life (HRQoL) is a multidimensional concept involving the subjective assessment of various dimensions of an individual's health status including physical function, mental or psychological status, bodily pain, emotional wellbeing, functional capacity, occupational function, and social integration [11].

There is currently no one definition for sarcopenia. Since 2010, there has been six consensus papers published [1,12-16], of which it appears that the European Working Group on Sarcopenia in Older People (EWGSOP) [1] definition is the most widely utilised. Generally speaking, there are two key elements to the diagnosis of sarcopenia: the presence of a low appendicular skeletal muscle mass (ASM) and either low grip strength or low muscle performance.

Both the EWGSOP [1] and the Asian Working Group on Sarcopenia (AWGS) [15] have proposed that quality of life should be one of the primary outcomes for future sarcopenia intervention trials. We have recently reported that the impact of sarcopenia on quality of life in older people has not been sufficiently explored, especially through prospective observational research [17]. To date, two cross sectional

*Correspondence to: Solomon Yu, National Health and Medical Council Centre of Research Excellence: Trans-Disciplinary Frailty Research to Achieve Health Aging, Australia, E-mail: Solomon.Yu@sa.gov.au

Key words: muscle mass, grip strength, muscle performance

Received: May 21, 2018; Accepted: June 06, 2018; Published: June 09, 2018 
studies investigating the association between sarcopenia and poor HRQoL in community dwelling older people have used the EWGSOP criteria $[18,19]$. There is a case for more research.

Our research aims to address this knowledge gap and the aim of our study is to assess the impact of sarcopenia (as defined by low muscle mass and low grip strength) on quality of life in community dwelling older South Australians, and also to determine whether there is any gender difference for this condition.

\section{Methods}

\section{Study design and participants}

This study had ethics approval from the Central Northern Adelaide Health Service Ethics of Human Research Committee (approval number: HREC/14/TQEH/276).

This is a cross-sectional study of community dwelling subjects aged 65 years and older from Stage 2 (2002-2004) of North West Adelaide Health Study (NWAHS). The characteristic of the NWAHS cohort and the recruitment method had been described in detail elsewhere but is briefly discussed here [20].

All households in the north-western area of Adelaide with a telephone connected and the telephone number listed in the Electronic White Pages were randomly selected for the study. The participants responded to a telephone interview conducted using computer-assisted telephone interview (CATI) technology, as well as completing a selfadministered questionnaire. Both the interview and questionnaire covered a broad range of issues including HRQoL, socio-demographic profile, medical and mental health problems, and risk factors.

4060 adults aged 18 years and older were recruited and underwent baseline biomedical examination between December 1999 and July 2003 (Stage 1). 3566 subjects participated in the follow-up study at 4 years, between May 2004 and February 2006 (Stage 2). In Stage 2, 3206 participants who had completed the interview and questionnaire attended the clinic for biomedical examination and investigations. This included measurements of blood pressure, anthropometric characteristics, grip strength, spirometry, and blood tests. Participants aged 50 years and over were invited to participate in the dual $x$-ray absorptiometry (DXA) investigation for osteoporosis. This current study included only Stage 2 participants who were 65 years and older with both dual absorptiometry x-ray (DXA) and grip strength measurements.

\section{Measurement of skeletal muscle mass}

In the NWAHS cohort, a Lunar PRODIGY scanner (GE Medical Systems, Madison, WI) in conjunction with Encore 2002 software was used to assess ASM. The skeletal muscle index (SMI) was calculated in order to take into account the differences in muscle mass due to height $\left(\mathrm{SMI}=\mathrm{ASM} /\right.$ height $\left.^{2}\right)$. In this current study, the cut-offs for low muscle mass was based on the gender specific lowest $20 \%$ of the SMI, which corresponds to a value of less than $7.34 \mathrm{~kg} / \mathrm{m}^{2}$ in men, and less than 5.80 $\mathrm{kg} / \mathrm{m}^{2}$ in women [9].

\section{Measurement of muscle strength}

Grip strength was assessed by a grip dynamometer (Lafayette Instrument Company, Lafayette, IN), with the subjects sitting and their arm supported by a horizontal surface. The maximum grip strength was calculated from the mean of three readings from the dominant hand [20]. Low grip strength was defined by a value less than $30 \mathrm{~kg}$ for males, and less than $20 \mathrm{~kg}$ for females [21]. These gender specific cut-off values for poor grip strength were used as these values had been shown to correspond to a low gait speed of less than $0.8 \mathrm{~m} / \mathrm{s}$ [22].

\section{Definition of sarcopenia}

Sarcopenia was defined as the presence of low muscle mass in conjunction with low hand grip strength [1].

\section{Measurement of HRQoL}

The participants HRQoL was assessed using the generic SF-36 quality of life (QoL) instrument [23]. The SF-36 is a self-reported health questionnaire, consisting of 36 items, which is designed to assess an individual's perceived health status across eight domains: Physical Functioning (PF), Role Physical (RP), Bodily Pain (BP), General Health (GH), Vitality (VT), Social Functioning (SF), Role Emotional (RE), and Mental Health (MH). From these eight health domains, two summary scores are derived which are the Physical Component Summary (PCS) scores (aggregate scores for PF, RP, GH, and BP), and the Mental Component Summary (MCS) scores (aggregate scores for VT, SF, RE, and $\mathrm{MH}$ ). Categorical response options are used for all the items. The responses to each item within a domain are summated to generate a score between $0-100$, with lower scores corresponding to poorer health status.

\section{Statistical analysis}

Analyses were carried out for all subjects as well as for males and females separately. Data were reported as means and standard deviations (SD) for continuous variables and relative frequencies for categorical variables. For continuous variables (i.e. SF-36 QoL scores), differences in mean values between sarcopenic and non-sarcopenic groups were assessed by an independent samples $t$-test. Chi-square test was utilised for nominal variables (co-morbidities and risk factors). The participants' co-morbidities were categorised using the Charlson Comorbidity Index (CCI) [24], which is a measure of an individual's disease burden. Multiple regression analysis was conducted to assess the association between the SF-36 quality of life domains with sarcopenia, with and without adjustment for confounding factors. Confounding factors included age, physical activity level, smoking status, CCI (score of one or more), and depression. SPSS 19 for Windows software (SPSS, Inc, Chicago, IL) was used for statistical analysis. A $P$ - value of less than 0.05 was considered statistically significant.

\section{Results}

727 subjects (out of 945 subjects from Stage 2; 357 males) aged 65 years and older had both grip strength and DXA results at stage 2 (Table 1, Supplementary Table S1). Males and females were similar age; $73.9 \pm 6.2$ and $73.2 \pm 6.0$ years old respectively $(P=0.097)$. The prevalence of sarcopenia was $10.1 \%$ in males and $9.5 \%$ in females. For both genders, subjects with sarcopenia were significantly $($ all $<0.001)$ older (79.0 \pm 6.3 vs. $73.4 \pm 5.9$ years in males, and $76.9 \pm 6.5$ vs. $72.8 \pm$ 5.9 years in females), had a lower body mass index $\left(24.1 \pm 3.0 \mathrm{~kg} / \mathrm{m}^{2} \mathrm{vs}\right.$. $28.1 \pm 3.9 \mathrm{~kg} / \mathrm{m}^{2}$ in males, and $23.4 \pm 3.6 \mathrm{~kg} / \mathrm{m}^{2}$ vs. $28.3 \pm 4.6 \mathrm{~kg} / \mathrm{m}^{2}$ in females), and had a smaller waist circumference $(93.3 \pm 9.9 \mathrm{~cm}$ vs. 101.8 $\pm 10.7 \mathrm{~cm}$ in males, and $83.6 \pm 11.4 \mathrm{~cm}$ vs. $91.6 \pm 11.3 \mathrm{~cm}$ in females), compared to non-sarcopenic subjects. In both genders, there were no significant differences in the prevalence of various co-morbidities and risk factors between sarcopenic and non-sarcopenic groups.

Univariate analyses of the various SF-36 quality of life domains for sarcopenic and non-sarcopenic subjects are presented in Table 2 and Supplementary Table S2. In all subjects, sarcopenic individuals had 
Table 1. Baseline characteristics of subjects

\begin{tabular}{|c|c|c|c|c|c|c|}
\hline & \multicolumn{3}{|c|}{ Male } & \multicolumn{3}{|c|}{ Female } \\
\hline & $\begin{array}{l}\text { Sarcopenia } \\
\quad(n=36)\end{array}$ & $\begin{array}{l}\text { No sarcopenia } \\
\quad(\mathbf{n}=\mathbf{3 2 1})\end{array}$ & $P$ - value & $\begin{array}{l}\text { Sarcopenia } \\
\quad(\mathrm{n}=\mathbf{3 5})\end{array}$ & $\begin{array}{l}\text { No sarcopenia } \\
\quad(\mathbf{n}=\mathbf{3 3 5})\end{array}$ & $P$-value \\
\hline \multicolumn{7}{|l|}{ Anthropometry Mean (SD) } \\
\hline Age (years) & $79.0(6.3)$ & $73.4(5.9)$ & $<0.001$ & $76.9(6.5)$ & $72.8(5.9)$ & $<0.001$ \\
\hline BMI $\left(\mathrm{kg} / \mathrm{m}^{2}\right)$ & $24.1(3.0)$ & $28.1(3.9)$ & $<0.001$ & $23.4(3.6)$ & $28.3(4.6)$ & $<0.001$ \\
\hline Waist circumference $(\mathrm{cm})$ & $93.3(9.9)$ & $101.8(10.7)$ & $<0.001$ & $83.6(11.4)$ & $91.6(11.3)$ & $<0.001$ \\
\hline ASM $/$ height $^{2}\left(\mathrm{~kg} / \mathrm{m}^{2}\right)$ & $6.93(0.33)$ & $8.22(0.78)$ & $<0.001$ & $5.43(0.33)$ & $6.52(0.72)$ & $<0.001$ \\
\hline Grip strength (kg) & $25.0(4.4)$ & $35.3(8.4)$ & $<0.001$ & $15.1(3.7)$ & $21.3(6.4)$ & $<0.001$ \\
\hline \multicolumn{7}{|c|}{ Co-morbidities and risk factors \% (n) } \\
\hline Cardiovascular disease & $44.4 \%(16)$ & $30.1 \%(95)$ & 0.078 & $27.3 \%(9)$ & $35.5 \%(117)$ & 0.347 \\
\hline Depression & $9.7 \%(3)$ & $5.3 \%(15)$ & 0.404 & $15.4 \%(4)$ & $8.9 \%(25)$ & 0.287 \\
\hline \multicolumn{7}{|l|}{ Physical activity level } \\
\hline Sedentary & $34.6 \%(9)$ & $30.0 \%(76)$ & \multirow{4}{*}{0.795} & $58.6 \%(17)$ & $40.8 \%(107)$ & \multirow{4}{*}{0.253} \\
\hline Low & $23.1 \%(6)$ & $29.6 \%(75)$ & & $24.1 \%(7)$ & $29.4 \%(77)$ & \\
\hline Moderate & $30.8 \%(8)$ & $32.8 \%(83)$ & & $17.2 \%(5)$ & $26.0 \%(68)$ & \\
\hline High & $11.5 \%(3)$ & $7.5 \%(19)$ & & $0.0 \%$ & $3.8 \%(10)$ & \\
\hline
\end{tabular}

BMI: Body mass index, ASM: Appendicular skeletal muscle mass

Table 2. Univariate analysis of SF-36 quality of life domains for men and women

\begin{tabular}{|c|c|c|c|c|c|c|}
\hline & \multicolumn{3}{|c|}{ Male } & \multicolumn{3}{|c|}{ Female } \\
\hline & $\begin{array}{l}\text { Sarcopenia } \\
\quad(\mathbf{n}=\mathbf{3 6})\end{array}$ & $\begin{array}{c}\text { No sarcopenia } \\
\quad(\mathbf{n}=\mathbf{3 2 1})\end{array}$ & $P$ - value & $\begin{array}{l}\text { Sarcopenia } \\
\quad(\mathbf{n}=\mathbf{3 5})\end{array}$ & $\begin{array}{l}\text { No sarcopenia } \\
\quad(\mathbf{n}=\mathbf{3 3 5})\end{array}$ & $P$ - value \\
\hline \multicolumn{7}{|l|}{ SF-36 score* } \\
\hline Physical component summary score & $57.0(28.9)$ & $67.8(22.3)$ & 0.012 & $55.3(25.9)$ & $64.0(23.9)$ & 0.060 \\
\hline Physical function & $56.0(30.6)$ & $68.9(24.7)$ & 0.005 & $48.3(24.3)$ & $60.7(25.8)$ & 0.009 \\
\hline General health & $55.9(24.6)$ & $63.6(19.4)$ & 0.033 & $58.6(19.3)$ & $64.2(19.9)$ & 0.130 \\
\hline Mental component summary score & $72.4(24.7)$ & $81.8(16.0)$ & 0.002 & $71.2(20.3)$ & $78.1(19.5)$ & 0.051 \\
\hline
\end{tabular}

*Data reported as mean (standard deviation)

significantly lower quality of life scores in all of the SF-36 quality of life domains, including the PCS and MCS scores, compared to nonsarcopenic subjects. When the data was analysed according to gender, only men with sarcopenia had statistically lower PCS and MCS scores compared to non-sarcopenic men. There was no statistical difference in both PCS and MCS scores between sarcopenic and non-sarcopenic women, though there was a strong trend for sarcopenic women to have lower PCS and MCS scores. In males, the PCS scores for sarcopenic and non-sarcopenic subjects were $57.0 \pm 28.9$ and $67.8 \pm 22.3$ respectively $(P=0.012)$. The MCS scores in sarcopenic and non-sarcopenic men were $72.4 \pm 24.7$ and $81.8 \pm 16.0$ respectively $(P=0.002)$. The PCS scores for sarcopenic females were $55.3 \pm 25.9$ compared to $64.0 \pm$ 23.9 in non-sarcopenic females $(P=0.060)$. In females, MCS scores for sarcopenic and non-sarcopenic subjects were 71.2 \pm 20.3 and $78.1 \pm$ 19.5 respectively $(P=0.051)$.

Multiple regression analysis of the association between sarcopenia and SF-36 quality of life domains are presented in Table 3 as well as Supplementary Tables S3, S4, S5, and S6. Sarcopenia was not a significant predictor of poorer PCS scores in all subjects, including men and women, after adjustment for all confounding variables $(P=0.487$ in all subjects, $P=0.092$ in men, and $P=0.609$ in women). Sarcopenia was however, significantly associated with the MCS scores in all subjects and in men after adjustment for all confounding variables $(P=0.017$ in all subjects, $P=0.001$ in men). For men, sarcopenia remained a significant predictor of poor HRQoL in multiple SF-36 quality of life domains after adjustment for all confounding variables. However, in women, the association between sarcopenia and SF-36 quality of life was lost after adjustments were made for confounding factors (i.e. there may be other factors that might potentially explain the poor quality of life in females other than sarcopenia).

\section{Discussion}

The key findings from this research demonstrated a clear gender difference in the impact of sarcopenia (as defined by low muscle mass and low grip strength) on HRQoL in community dwelling older Australian people. After adjusting for multiple confounders, the association between sarcopenia only remained for the MCS score in men. No association between sarcopenia and HRQoL was seen in women. Even though our study did not find an association between sarcopenia and HRQoL in unadjusted model for females, there was however a strong trend for both PCS and MCS scores, with the MCS score almost reaching significance at a $P$ value of 0.051 in the unadjusted model.

To date, there have been two cross-sectional studies reporting on the relationship between sarcopenia including low ASM and at least one performance measure and SF-36 HRQoL $[18,19]$. Both the Patel and Beaudart study complied with the EWSGOP criteria in that sarcopenia was defined as low muscle mass and either low muscle strength or low muscle performance (i.e. gait speed or short physical performance battery score). In our study however, gait speed was not available, and we relied on the combination of low muscle mass and low grip strength and this difference in method may partially account for the difference in prevalence figures reported. Our study found that $10.1 \%$ of men and $9.5 \%$ of women were affected by sarcopenia. The cross-sectional study by Patel [18], which included 1787 community dwelling subjects in the United Kingdom demonstrated the prevalence of sarcopenia to be $4.6 \%$ in men and $7.9 \%$ in women but rather than DXA, skin fold thickness was used to assess muscle mass. Beaudart's study [19], which focused on 534 community dwelling subjects in Belgium, reported a prevalence of sarcopenia of $11.8 \%$ in men and $14.9 \%$ in women. In the Patel study, sarcopenia was associated with poorer general health $(\mathrm{GH})$ and physical 
Table 3. Relationship between sarcopenia and SF-36 quality of life in men and women

\begin{tabular}{|c|c|c|c|c|c|c|c|c|}
\hline & \multicolumn{4}{|c|}{ Male } & \multicolumn{4}{|c|}{ Female } \\
\hline & \multicolumn{2}{|c|}{ PCS } & \multicolumn{2}{|c|}{ MCS } & \multicolumn{2}{|c|}{ PCS } & \multicolumn{2}{|c|}{ MCS } \\
\hline & & $P$-value & & $P$-value & & $P$ - value & & $P$-value \\
\hline Unadjusted & $\begin{array}{l}\mathrm{B}=-10.81 \\
\mathrm{SE}_{\mathrm{B}}=4.29 \\
\beta=-0.138\end{array}$ & 0.012 & $\begin{array}{l}\mathrm{B}=-9.39 \\
\mathrm{SE}_{\mathrm{B}}=3.04 \\
\beta=-0.165\end{array}$ & 0.002 & $\begin{array}{l}\mathrm{B}=-8.71 \\
\mathrm{SE}_{\mathrm{B}}=4.61 \\
\beta=-0.103\end{array}$ & 0.060 & $\begin{array}{l}\mathrm{B}=-6.82 \\
\mathrm{SE}_{\mathrm{B}}=3.49 \\
\beta=-0.102\end{array}$ & 0.051 \\
\hline Model 1 & $\begin{array}{l}\mathrm{B}=-9.16 \\
\mathrm{SE}_{\mathrm{B}}=4.46 \\
\beta=-0.117\end{array}$ & 0.041 & $\begin{array}{l}\mathrm{B}=-10.59 \\
\mathrm{SE}_{\mathrm{B}}=3.17 \\
\beta=-0.186\end{array}$ & 0.001 & $\begin{array}{l}\mathrm{B}=-4.86 \\
\mathrm{SE}_{\mathrm{B}}=4.56 \\
\beta=-0.058\end{array}$ & 0.287 & $\begin{array}{l}\mathrm{B}=-4.75 \\
\mathrm{SE}_{\mathrm{B}}=3.52 \\
\beta=-0.071\end{array}$ & 0.178 \\
\hline Model 2 & $\begin{array}{l}\mathrm{B}=-9.19 \\
\mathrm{SE}_{\mathrm{B}}=5.20 \\
\beta=-0.112\end{array}$ & 0.078 & $\begin{array}{l}\mathrm{B}=-11.61 \\
\mathrm{SE}_{\mathrm{B}}=3.65 \\
\beta=-0.200\end{array}$ & 0.002 & $\begin{array}{l}\mathrm{B}=-1.12 \\
\mathrm{SE}_{\mathrm{B}}=4.94 \\
\beta=-0.013\end{array}$ & 0.821 & $\begin{array}{c}\mathrm{B}=-1.84 \\
\mathrm{SE}_{\mathrm{B}}=3.90 \\
\beta=-0.028\end{array}$ & 0.637 \\
\hline Model 3 & $\begin{array}{l}\mathrm{B}=-8.93 \\
\mathrm{SE}_{\mathrm{B}}=5.27 \\
\beta=-0.110\end{array}$ & 0.092 & $\begin{array}{l}\mathrm{B}=-11.60 \\
\mathrm{SE}_{\mathrm{B}}=3.30 \\
\beta=-0.202\end{array}$ & 0.001 & $\begin{array}{c}\mathrm{B}=3.24 \\
\mathrm{SE}_{\mathrm{B}}=6.32 \\
\beta=0.034\end{array}$ & 0.609 & $\begin{array}{c}\mathrm{B}=0.397 \\
\mathrm{SE}_{\mathrm{B}}=3.70 \\
\beta=0.006\end{array}$ & 0.915 \\
\hline
\end{tabular}

B: Unstandardized regression coefficient, SE : Standard error of the coefficient, $\beta$ : Standardized coefficient, PCS: physical component summary, MCS: mental component summary, Model 1: sarcopenia + age, Model 2: model $1+$ physical activity + smoking status, Model 3: model $2+$ Charlson Co-morbidity Index + depression

function (PF) scores in both men and women but no adjustment for confounding variables occurred. The Beaudart study did not report separately on men and women but noted that sarcopenic subjects had lower physical function scores than subjects with no sarcopenia. Similarly, our study also found an association between sarcopenia and PF scores in all subjects but unlike the Patel study where subjects were younger and muscle mass was assessed using skin fold thickness, this association was noted only in men and for both associations only when unadjusted for confounding variables. In the Beaudart study, no other association was noted between sarcopenia and any other domain of HRQOL. In contrast, we noted an association between sarcopenia and all measured domains for HRQOL (Supplementary Tables S3 and S4) and associations remained after adjustment for all confounding variables (i.e. model 3) for MCS, RE, and $\mathrm{MH}$ in all subjects. The Beaudart study utilised a different statistical method (i.e. logistic regression), adjusted for different co-variables (i.e. BMI, cognitive and nutritional status, number of medications) and as already discussed used different measures to define sarcopenia and collectively these might partially account for the differences in study findings.

Both the Patel and Beaudart study did not report on the composite PCS and MCS scores and in our study, all eight quality of life domains as well as the PCS and MCS scores of the SF-36 were included. The Patel study only investigated the association between sarcopenia and GH and PF domains in men and women. Our study demonstrated that after adjustment for confounders, only men with sarcopenia was significantly associated with poorer MCS, GH, and BP scores as well as poorer scores in all four mental health domains of the SF-36 (i.e. VT, RE, SF, and MH). We postulate several possible reasons to explain why the association between sarcopenia and HRQoL was lost after adjustment for confounders for women in our current study. This discrepancy in the quality of life results between males and females in our study might be explained by the fact that mediators in the relationship between strength and quality of life may be different between men and women $[25,26]$. In women, functional capacity (i.e. ability to perform instrumental activities of daily living) has been shown to mediate the relationship between strength and quality of life, while in men, strength is directly related to quality of life [25]. In the study by Beaudart [19], sarcopenic women were significantly more impaired for some aspects of the instrumental activities of daily living (IADL) such as housekeeping and ability to manage finances compared to non-sarcopenic women. In contrast, sarcopenic men do not seem to be more dependent in the various aspects of IADL compared with nonsarcopenic men [19]. There may also be other potential factors leading to functional impairment that accounted for the poor quality of life in women other than sarcopenia. Life style factors such as smoking and low physical activity had been shown to have a greater negative effect on HRQoL in women compared to men [27-29]. The Australian study by Jayasinghe [27] showed that female smokers had clinically significant poorer quality of life in the mental health domain compared to nonsmokers. Other studies had demonstrated that females with low or no physical activity were strong predictors of poorer quality of life $[28,29]$. Medical co-morbidities such as arthritis, diabetes, osteoporosis, and cardiovascular disease are also strongly associated with adverse HRQoL in women [28]. A study has shown that females who had stroke or transient ischaemic attack were significantly more negatively affected in their quality of life compared to their male counterparts [26], and that this may in part, be mediated by the functional disability after stroke [30].

There has only been one longitudinal study investigating the relationship between muscle performance and muscle strength and quality of life. The Bindawas' study [31] assessed the association between the short physical performance battery (SPPB) and muscle strength with the SF-36 quality of life in 621 community dwelling older Mexican Americans over a period of six years. SPPB and muscle strength were noted to be independent predictors of the SF-36 PCS score at six years for both genders [31]. In addition, high total muscle strength was significantly associated with slower rate of decline in the MCS scores over time. There is therefore a need for longitudinal evaluation of the relationship between sarcopenia and quality of life.

Even though the therapeutic role of nutritional intervention alone (i.e. protein supplementation) in the management of sarcopenia is inconsistent [32], it is postulated that the combined effects of exercise training and high quality nutritional supplementation, especially with protein supplementation, has a role in preventing and managing sarcopenia [33]. However, no studies to date have confirmed the benefit of exercise in combination with nutritional supplementation in improving quality of life for sarcopenic older people and this is an area of research that would benefit from attention [34,35].

There are several limitations to this study. Firstly, due to the community-based nature of this study, subjects from residential care facility or those who were frailer and home- bound were not assessed. 
The prevalence of sarcopenia and the likelihood of poorer quality of life are likely higher in these population groups. The subjects in this study were predominantly Caucasian and therefore not representative of the multi-cultural Australian society of today. Therefore, it is important that the findings of this study are confirmed in other population groups. It would have been ideal to be able to adjust for cognitive status as a confounding variable but this was not assessed in this study. Finally, as this was a cross-sectional study, the causal relationship between sarcopenia and quality of life could not be established.

\section{Conclusion}

This is the first study assessing the association of sarcopenia and HRQoL in older Australians. Although there have been two previous cross-sectional studies elsewhere on this topic, this study adds to the literature as it demonstrated a clear gender difference in regard to the impact of sarcopenia on HRQoL in men and women. After adjustment for multiple confounders, sarcopenia in men was an independent predictor of poorer quality of life. No association between sarcopenia and HRQoL was seen in women. It is important to undertake longitudinal analysis to determine if sarcopenia predicts poor quality of life in older people. It is equally necessary to investigate if interventions such as physical activity or protein supplementation can improve quality of life in those with sarcopenia.

\section{Conflict of interest}

- Dr Tsung Woo has nothing to declare.

- Assoc. Prof Solomon Yu has nothing to declare.

- Professor Robert Adams has nothing to declare.

- Professor Renuka Visvanathan is on the malnutrition in the elderly board, Nestle Australia and has participated in international initiatives (i.e. PROTAGE and MNA Initiative) made possible by educational grants by Nestle Inc. In the past, Professor Visvanathan has received educational and research grant funding from Organon Pty Ltd, Servier, Novartis and Pfizer.

\section{References}

1. Cruz-Jentoft AJ, Baeyens JP, Bauer JM, Boirie Y, Cederholm T, et al. (2010) Sarcopenia: European consensus on definition and diagnosis: Report of the European Working Group on Sarcopenia in Older People. Age Ageing 39: 412-423. [Crossref]

2. Walston JD (2012) Sarcopenia in older adults. Curr Opin Rheumatol 24: 623-627. [Crossref]

3. Guralnik JM, Ferrucci L, Pieper CF (2000) Lower extremity function and subsequent disability: consistency across studies, predictive models, and value of gait speed alone compared with the short physical performance battery. J Gerontol A Biol Sci Med Sci 55: M221-M31. [Crossref]

4. Rantanen T, Guralnik JM, Foley D, Masaki K, Leveille S, et al. (1999) Midlife hand grip strength as a predictor of old age disability. JAMA 281: 558-560. [Crossref]

5. Landi F, Cruz-Jentoft AJ, Liperoti R, Russo A, Giovannini S, et al. (2013) Sarcopenia and mortality risk in frail older persons aged 80 years and older: results from ilSIRENTE study. Age Ageing 42: 203-209. [Crossref]

6. Arango-Lopera VE, Arroyo P, Gutiérrez-Robledo LM, Pérez-Zepeda MU, Cesari M (2013) Mortality as an adverse outcome of sarcopenia. J Nutr Health Aging 17: 259262. [Crossref]

7. Cooper R, Kuh D, Cooper C, Gale CR, Lawlor DA, et al. (2011) Objective measures of physical capability and subsequent health: a systematic review. Age Ageing 40: 14-23. [Crossref]

8. Janssen I, Shepard DS, Katzmarzyk PT, Roubenoff R (2004) The healthcare costs of sarcopenia in the United States. J Am Geriatr Soc 52: 80-85. [Crossref]
9. Yu S, Appleton S, Adams R, Chapman I, Wittert G, et al. (2014) The impact of low muscle mass definition on the prevalence of sarcopenia in older Australians. Biomed Res Int 2014: 361790. [Crossref]

10. Hickey A, Barker M, McGee H, O’Boyle C (2005) Measuring health-related quality of life in older patient populations: a review of current approaches. Pharmacoeconomics 23: 971-993.

11. Rizzoli R, Reginster JY, Arnal JF, Bautmans I, Beaudart C, et al. (2013) Quality of life in sarcopenia and frailty. Calcif Tissue Int 93: 101-120. [Crossref]

12. Fielding RA, Vellas B, Evans WJ (2011) Sarcopenia: an undiagnosed condition in older adults. Current consensus definition: prevalence, etiology, and consequences. International working group on sarcopenia. J Am Med Dir Assoc 12(4):249-56. [Crossref]

13. Muscaritoli M, Anker SD, Argiles J (2010) Consensus definition of sarcopenia, cachexia and pre-cachexia: joint document elaborated by Special Interest Groups (SIG) "cachexia-anorexia in chronic wasting diseases" and "nutrition in geriatrics". Clinical nutrition 29:154-159. [Crossref]

14. Morley JE, Abbatecola AM, Argiles JM (2011) Sarcopenia with limited mobility: an international consensus. J Am Med Dir Assoc 12(6):403-9. [Crossref]

15. Chen LK, Liu LK, Woo J (2014) Sarcopenia in Asia: consensus report of the asian working group for sarcopenia. J Am Med Dir Assoc 15: 95-101. [Crossref]

16. Studenski SA, Peters KW, Alley DE, Cawthon PM, McLean RR, et al. (2014) The FNIH sarcopenia project: rationale, study description, conference recommendations, and final estimates. J Gerontol A Biol Sci Med Sci 69: 547-558. [Crossref]

17. Woo T, Yu S, Visvanathan R (2016) Systematic literature review on the relationship between biomarkers of sarcopenia and quality of life in older people. J Frailty Aging 5: 88-99.

18. Patel HP, Syddall HE, Jameson K (2013) Prevalence of sarcopenia in communitydwelling older people in the UK using the European Working Group on Sarcopenia in Older People (EWGSOP) definition: findings from the Hertfordshire Cohort Study (HCS). Age and ageing 42: 378-84. [Crossref]

19. Beaudart C, Reginster JY, Petermans J, Gillain S, Quabron A, et al. (2015) Quality of life and physical components linked to sarcopenia: The SarcoPhAge study. Exp Gerontol 69: 103-110. [Crossref]

20. Grant JF, Chittleborough CR, Taylor AW (2006) The North West Adelaide Health Study: Detailed methods and baseline segmentation of a cohort for selected chronic diseases. Epidemiol Perpect Innov 3. [Crossref]

21. Lauretani F, Russo CR, Bandinelli S, Bartali B, Cavazzini C, et al. (2003) Ageassociated changes in skeletal muscles and their effect on mobility: an operational diagnosis of sarcopenia. J Appl Physiol (1985) 95: 1851-1860. [Crossref]

22. Ware JE (1997) SF-36 Health Survey. Manual and Interpretation Guide (2nd Edition) Nimrod Press, MA, USA: The Health Institute, New England Medical Centre.

23. Charlson ME, Pompei P, Ales KL, MacKenzie CR (1987) A new method of classifying prognostic comorbidity in longitudinal studies: development and validation. J Chronic Dis 40: 373-383. [Crossref]

24. Benton MJ, Alexander JL, Holland JD (2014) Relationship between strength, function, and quality of life in older adults with chronic lung disease: is there an influence of gender? J Cardiopulm Rehabil Prev 34: 143-149. [Crossref]

25. Franzén-Dahlin Å, Laska AC (2012) Gender differences in quality of life after stroke and TIA: a cross-sectional survey of out-patients. J Clin Nurs 21: 2386-2391. [Crossref]

26. Jayasinghe UW, Harris MF, Taggart J, Christl B, Black DA (2013) Gender difference in health-related quality of life of Australian chronically-ill adults: patient and physician characteristics do matter. Health Qual Life Outcomes 11: 1-12.

27. Dale CE, Bowling A, Adamson J (2013) Predictors of patterns of change in healthrelated quality of life in older women over 7 years: evidence from a prospective cohort study. Age and ageing 42: 312-318. [Crossref]

28. Campos ACV, Ferreira EFe, Vargas AMD, Albala C. Aging, Gender and Quality of Life (AGEQOL) study: factors associated with good quality of life in older Brazilian communty-dwelling adults. Health Qual Life Outcomes. 12: 1-11. [Crossref]

29. Sturm JW, Donnan GA, Dewey HM, Macdonell RA, Gilligan AK, et al. (2004) Quality of life after stroke: the North East Melbourne Stroke Incidence Study (NEMESIS). Stroke 35: 2340-2345. [Crossref]

30. Bindawas SM, Al Snih S, Ottenbacher AJ (2015) Association Between Lower Extremity Performance and Health-Related Quality of Life in Elderly Mexican Americans. $J$ Aging Health 27: 1026-1045. [Crossref] 
Woo T (2018) The association between sarcopenia and quality of life is different in community dwelling older Australian men and women

31. Cruz-Jentoft AJ, Landi F, Schneider SM (2014) Prevalence of and interventions for sarcopenia in ageing adults: a systematic review. Report of the International Sarcopenia Initiative (EWGSOP and IWGS). Age and ageing. 43: 748-759. [Crossref]

32. Denison HJ, Cooper C, Sayer AA, Robinson SM (2015) Prevention and optimal management of sarcopenia: a review of combined exercise and nutrition interventions to improve muscle outcomes in older people. Clin Interv Aging 10: 859-869. [Crossref]
33. Chou CH, Hwang CL, Wu YT (2012) Effect of exercise on physical function, daily living activities, and quality of life in the frail older adults: a meta-analysis. Arch Phys Med Rehabil 93: 237-244. [Crossref]

34. Langlois F, Vu TT, Chassé K, Dupuis G, Kergoat MJ, et al. (2013) Benefits of physical exercise training on cognition and quality of life in frail older adults. $J$ Gerontol B Psychol Sci Soc Sci 68: 400-404. [Crossref]

Copyright: $(2018$ Woo T. This is an open-access article distributed under the terms of the Creative Commons Attribution License, which permits unrestricted use, distribution, and reproduction in any medium, provided the original author and source are credited. 